\title{
Development and Evaluation of an Indirect ELISA for Detection of Infection with Teladorsagia Circumcincta in Sheep
}

Jalal Aliabadi

Shiraz University

Ehsan Rakhshandehroo ( $\sim$ Rakhshandehroo@Shirazu.ac.ir)

Shiraz University

Azadeh Yektaseresht

Shiraz University

\section{Research Article}

Keywords: Teladorsagia circumcincta, Western blotting, ELISA, Sheep.

Posted Date: April 7th, 2021

DOI: https://doi.org/10.21203/rs.3.rs-386999/v1

License: (c) (i) This work is licensed under a Creative Commons Attribution 4.0 International License.

Read Full License 


\section{Abstract \\ Background}

The gastrointestinal helminth, Teladorsagia circumcincta, is one of the major health risk and productionlimiting disease in small ruminant populations, particularly in temperate regions. With the increasing importance of the disease management and recruited anthelminic resistant types, accurate approaches are needed for diagnosis of the infection in the host. Because of uncertain results using faecal examinations, the ELISA method was indicated for detection of the nematode antigenic materials. Despite some promising results, problems were described in terms of the test specificity and the crossreactions. The aim of this study was therefore to evaluate worm somatic and excretory/secretory (ES) products using western blot analysis and an indirect ELISA for detecting T. circumcincta infection in sheep.

\section{Results}

Based on the immuno-reactivity analysis, immunogenic fractions with molecular weights of approximately 60, 75 and $100 \mathrm{kDa}$ were detected in somatic content and two antigens of about 63 and $75 \mathrm{kDa}$ in ES material. Accordingly, a specific product at $75 \mathrm{kDa}$ had the strongest reaction and appeared as the most common antigenic protein. In ELISA, all sera from the infected sheep revealed the OD rates above the calculated cut-off value with about two-fold greater in average. Negative control samples were also specifically recognized with the mean OD rate of about $1 / 3$ of the estimated cut-off value. The crossreaction test, using rabbit hyperimmune sera, did not show reactivity with ES antigens of other prevalent nematodes include Haemonchus contortus, Protostrongylus rufescens and Marshallagia marshalli. In contrast, a strong positive reaction was found with the somatic antigens of $M$. marshalli.

\section{Conclusions}

The results obtained here indicates the indirect ELISA method using the ES content enables distinguishing the T. circumcincta infected sheep with high specificity. Those antigenic ES peptides with 63 and particularly $75 \mathrm{kDa}$ molecular weights should be more investigated due to the potential for serological diagnostic methods and immunoprotective targets in the host.

\section{Background}

Teladorsagia circumcincta is one of the most prevalent gastrointestinal nematode (GIN) which infects sheep and goats worldwide. The infection coincides with inflammation in the abomasal tissue leading to changes in the gastric physiological functions [1] and contributes to reduced weight gain and productive indicators [2]. 
Detection of the GIN has been traditionally depend on tracing the eggs in faecal samples by microscopy. Several egg count procedures with subsequent modifications have reported to estimate the level of infection. However, microscopic assessments have some disadvantages and often accompanied with unreliable results [3]. Therefore, alternative diagnostic approaches were developed to identify the present GIN infections in the host. Among the evaluated methods, the response of the immune system have been mostly investigated [4]. In sheep infected with $T$. circumcincta, protective immunity was associated with the parasite-specific antibodies against adult worms [5] and the established larvae [6, 7]. Isotypes of antibodies play important roles in GIN resistance in sheep, including $\lg A$, IgG1 and IgE [8].

In the past, potential of the ELISA technique was assessed to find out the antibody response to Ostertagia species in field applications. The IgA reaction comparatively investigated in larval somatic antigen and a fragment of a recombinant protein, disulphide isomerase, in blood, nasal secretions and saliva of the infected ewes [9]. This method was also developed for detection of IgG antibodies against copro-antigens in faecal preparations [10]. Additionally, the level of IgG specific to T. circumcincta crude antigens described in milk and blood of goats [11] and experimentally infected lactating ewes [12]. In cattle, ELISA was hopefully performed for diagnosis of gut-associated nematode infections using recombinant Cooperia oncophora protein [13], crude Ostertagia ostertagi whole-antigen [14] and Ostertagia ostertagi copro-antigens containing excretory secretory (ES) products of the worm [15]. Alongside with the successful or relatively promising results, some studies reported the strong cross-reactions with other nematode antigens among the trichostrongyloid members [16] and the difficulty to obtain crude [13] or somatic antigens [9] with highly standardized preparations. More importantly, improvements may also need to enhance the test sensivity for the parasite detection [10].

Because of variations in or lack of data on test specificity, more investigations are needed to enhance diagnosis based on tracing antigens of T. circumcincta in the host. Therefore, the objective of this study was first to detect and characterize the somatic and ES antigens of T. circumcincta in sheep. In addition, those antigens were used to develop a specific ELISA method with high sensivity or specificity rates. In parallel, the possibility of cross-reactions was evaluated with some prevalent nematodes that are usually found in abomasal and lung tissues.

\section{Results}

\section{Species confirmation}

In this study, data from molecular evaluations corroborated morphologic diagnosis. The BLAST search indicated major similarities between the sequence data obtained (MN888739) and available reports for $T$. circumcincta (The phylogenetic relations was depicted in Additional file 1).

\section{SDS-PAGE and western blot Analysis}

Total proteins of somatic and ES antigens were determined as $22.1 \mathrm{mg} / \mathrm{ml}$ and $15 \mathrm{mg} / \mathrm{ml}$, respectively. The SDS-PAGE analysis for somatic antigens revealed 15 protein fractions ranging in size from 20 to 245 
$\mathrm{kDa}$, with molecular weights (MW) of 20, 25, 30, 38, 42 (weak band), 45, 47, 60, 63, 65, 75, 80, 100, 180, $245 \mathrm{kDa}$ (Fig. 2). In the pattern of ES antigens, proteins with MWs of 20, 25 (weak band), 28, 35, 48, 50, $63,68,75,80,100,135,180 \mathrm{kDa}$ could be detected (Fig. 2).

The immuno-reactivity of the somatic antigens with sera from the infected sheep revealed three proteins of about 60,75 and $100 \mathrm{kDa}$ (Fig. 3A). However, with the hyper immune serum of rabbits, the result was fractions with MWs of about 30,60 and $100 \mathrm{kDa}$ (Fig. 4). The immunoblotting analysis with ES antigens and positive sera of sheep demonstrated only two bands of 63 and $75 \mathrm{kDa}$ (Fig. 3A). One specific band at $75 \mathrm{kDa}$ had the strongest reaction (Fig. 3B). In comparison, fractions with 50, 75 and $135 \mathrm{kDa}$ were obtained using sera of the immunized rabbits (Fig. 3B). Based on the present electrophoretic patterns, the immune response to somatic antigens of 60 and $100 \mathrm{kDa}$ and $\mathrm{ES}$ antigen with $75 \mathrm{kDa}$ were commonly found in both animal types. In addition, among the somatic and ES protein profiles, the product with 75 $\mathrm{kDa} \mathrm{MW}$ was the most common antigenic protein. Immunoblotting analysis gave negative reactions with sera from non-infected sheep.

On Western blot analysis, the specificity of rabbit sera against $T$. circumcincta ES antigens was confirmed by lack of any cross reaction with $H$. contortus, $P$. rufescens and $M$. marshalli ES materials. In contrast, positive anti-somatic sera revealed strong reactivities with the somatic antigens of M. marshalli at 35, 63, 75 and $100 \mathrm{kDa}$. A slight reaction was also found against somatic antigens of H. contortus (Fig. 4).

\section{ELISA}

The ODR values obtained with serum samples of the worm-free (negative) and positive samples against the somatic and ES antigens are plotted in Fig. 5. The Mean \pm SEM of ODR values were $0.708 \pm 0.03$ for positive samples against somatic and $0.674 \pm 0.02$ for ES antigens. The respective cut-off values were calculated as 0.348 and 0.328 . All the samples from the infected sheep indicated ODR values above the cut-off thresholds. In addition, negative samples registered ODRs with overall Mean $\pm S E M$ of $0.12 \pm 0.014$ ranged 0.038 to 0.215 . This means that the present antigens gave a $100 \%$ sensivity and $100 \%$ specificity for diagnosis of the infection.

According to observed reactions and the ODR values obtained, no cross-reactions were detected between T. circumcincta somatic and ES antigens against the same for $H$. contortus, P. rufescens and ES from $M$. marshalli. As expected before, the relatively high ODR value confirmed a significant reaction with somatic antigens of M. marshalli (Fig. 6).

\section{Discussion}

Based on the results obtained in this study, the ELISA test using the somatic and ES antigens is a sensitive approach for detection of the infection with $T$. circumcincta in sheep. Compared with somatic antigens, the ES products was more specific with no cross reactivity with some other prevalent GIN nematode. Among ES antigens, a peptide with $75 \mathrm{kDa}$ molecular weight was commonly identified in 
different specimens with negative cross reactions indicating the potential for serological diagnostic methods and immunoprotective studies in future.

The role of somatic and ES materials has been defined according to the host immune response and protection against GIN infections in ruminants. Many of secreted proteins, particularly from the ES components, have been characterized in larval and adult stages of trichostrongylid worms (reviewed in [24]). Studies are more directed on the larval secreted proteins with protective potential and applied them for vaccine development [25]. A previous research on adult T. circumcincta ES products reported peptides varying from 18 to $115 \mathrm{kDa}$ with the most prominent proteins at 20,29 and $63 \mathrm{kDa}$ molecular weights [26]. These proteins, which were also indicated in our results, are homologues of enzymes involved in energy metabolism reported in Ostertagia ostertagi and $C$. elegans. In line with our data, a major protein with $35 \mathrm{kDa} \mathrm{MW}$ and a relatively weak $25 \mathrm{kDa} \mathrm{MW}$ protein band were described in the ES products of fourth stage larva with Cathepsin F function (TCi-CF-1) [27]. The corresponding CF was also explained previously as the most abundant protein with about 27 to $30 \mathrm{kDa}$ molecule in larval ES from $T$. circumcincta [28]. Despite some similarities, other proteins in the adult stage ES with MWs of about 75 $\mathrm{kDa}$ and higher and the whole somatic extract of T. circumcincta were not described earlier. Nevertheless, according to the immunoblotting data here, ES proteins with MWs of 63 and $75 \mathrm{kDa}$ and somatic antigens with 60, 75 and $100 \mathrm{kDa}$ MW could be considered for immunization against and detection of the infection with $T$. circumcincta in sheep.

In this study, the explained indirect ES ELISA was a promising specific method to distinguish between $T$. circumcincta and some other prevalent nematode infections. The cross-reaction among trichostrongylid nematodes has been a limiting factor for test specificity. Levels of reactivities were reported between somatic $[11,16]$ and ES $[10,29,30]$ products derived from T. circumcincta, $H$. contortus, Trichostrongylys colubriformis and a trematode, Fasciola hepatica. Therefore, improvements in the method applied [10], application of purified somatic antigens [31] and using specific recombinant antigens [24] were suggested to overcome this major problem. However, because of some partial reactivities, the differentiation between $T$. circumcincta and $H$. contortus was remained to be solved. An improved coproantigen ES ELISA was evaluated for detection of $T$. circumcincta infection in faecal samples in sheep [10]. A great accuracy was obtained and the optimized period of exposure to an optimum temperature largely reduced the cross-reactive signals from all but two cases of $H$. contortus. In another research, the hyperimmune sera against soluble extract of T. circumcincta recognized some protein fractions of $H$. contortus except for a $26 \mathrm{kDa}$ peptide, which was exclusively reactive with homologus antiserum [31]. Such common antigens have more reported for somatic antigens [30]. In addition to more sensitive results, the ES content acts more specifically to detect $H$. contortus infections [16]. In our study, a faint reaction was recognized in immunoblotting assay between somatic (but not ES) content of $H$. contortus and T. circumcincta. Fortunately, this observation did not confirmed in the ELISA method. In comparison, the significant reactivity was only found against somatic antigens of $M$. marshalli. This result could be expected mainly due to the close phylogenetic relation between Marshallagia and Ostertagia, both belonged to Ostertagid nematodes. This is worthy of note that we did not access to sera from sheep (experimentally or naturally) mono-infected with prevalent GIN and thereby the immunized rabbit sera 
was substituted. Thus, the assay needs more evaluated using sera samples from the infected sheep from different regions.

The primary aim for the ELISA test was developing a feasible and specific assay. The ODR value seems to be a reliable index to for animals with moderate infections (with about 1000 or more adult worms) with need to the anthelmintic treatments. However, those animals with low or subacute chronic infections should be of concern. This issue was previously considered on ES materials in faecal samples from sheep infected with fewer than 1000 T. circumcincta, which was coincided with variable and overlapped OD values relative to the calculated threshold [10]. Similar unsatisfied results was also reported by ELISA on faecal $O$. ostertagi ES content in cattle with natural or experimental infections [15]. In this issue, even if it is postulated that the ELISA would not discriminate sera of sheep with low infection intensities and negative controls, our study contributes to show the practical advantage of this method in line with targeted (selective) treatment [32] to detect and treat those animals harboring much heavier infections.

\section{Conclusions}

This study concluded the functional value of the ELISA test with ES products for screening of infection with T. circumcincta in sheep. A strong cross-reaction was observed with somatic antigens of $M$. marshalli, while this reaction was not the case for ES products. According to immunoblot analysis, purified antigenic fractions (like those with MWs of about 63 and $75 \mathrm{kDa}$ ) is suggested to be investigated in a broader number of infected and non-infected sheep and goats.

\section{Methods}

\section{Collection of worm and blood samples}

A total of forty sheep (between 1-2 years old), with relatively high abomasal infections (harbored about 1000 to 2000 worms), were selected randomly from different flocks referred to the local industrial abattoirs in Shiraz $\left(29.5926^{\circ} \mathrm{N}, 52.5836^{\circ} \mathrm{E}\right)$, Fars province, South of Iran. The abomasa cut and placed in warm $37^{\circ} \mathrm{C}$ phosphate-buffered saline (PBS). Worms were then recovered, washed three times in PBS, pH 7.2 , containing penicillin $(10000 \mathrm{lU} / \mathrm{ml})$ and streptomycin $(10 \mathrm{mg} / \mathrm{ml})$ and used for antigen extraction. A number of worms were morphologically identified according to previous descriptions for T. circumcincta [17]. Regarding the prevalence of other trichostrongylid nematodes in the sampled area, efforts were made to ensure full recovery of worm burden to exclude abomasa with mixed infection. Prior to the slaughtering process, peripheral whole blood samples were collected from the jugular vein of animals. Sera were separated from the samples collected from the infected sheep and maintained at $-20^{\circ} \mathrm{C}$ until use. In addition, blood samples from inspected 10 non-infected animals (under 6 months of age) with no nematode infection were sampled as negative controls. Sample collections were performed after the consent to participate was obtained from farm owners.

\section{Molecular diagnosis}


Adult $T$. circumcincta worms were randomly selected from at least 2 farms and subjected to genomic extraction using a DNA extraction Kit (MBST, Iran). A primer set was used: $\mathrm{F}$ ( $5^{\prime}$ -

GCAGACGCTTAGAGTGGTGA-3') and R (5'-TCCTTGTTAGTTTCTTTTCCTCCG-3'), as described previously to identify the complete rDNA ITS-2 region in ostertagiine nematodes [18]. The PCR reaction mix included $12.5 \mu \mathrm{l}$ of PCR premix (Ampliqon, Denmark, Cat. No. A180301), $1 \mu \mathrm{l}$ of each primer, $6.5 \mu \mathrm{l} \mathrm{H}_{2} \mathrm{O}$ and $4 \mu \mathrm{l}$ of DNA as template. The cycling program consisted of an initial denaturation at $95^{\circ} \mathrm{C}$ for $5 \mathrm{~min}, 94^{\circ} \mathrm{C}$ for 30 sec, followed by $35 \mathrm{cycles}$ of $60^{\circ} \mathrm{C}$ for $30 \mathrm{sec}$, the extension at $72^{\circ} \mathrm{C}$ for $30 \mathrm{sec}$ and the final extension at $72^{\circ} \mathrm{C}$ for $10 \mathrm{~min}$. The products were sequenced (ABI 3730 DNA analyzer; Bioneer, Korea) and compared with other available sequences in NCBI using BLAST search. The sequence data was aligned with homologous sequences existing in the GenBank using Clustal W program by M EGA 6 software [19]. The phylogenetic tree was constructed by maximum likelihood $(\mathrm{ML})$ method and analyses was carried out using the Kimura 2-parameter distance estimate [20].

\section{Preparation of T. circumcincta somatic and ES antigens}

Somatic antigens were extracted from washed adult specimens. A total of (about) 1000 worms were separately homogenized by tissue grinding, sonicated in $10 \mathrm{~mL} \mathrm{PBS} \mathrm{pH} 7.2$ and centrifuged at $12000 \mathrm{rpm}$ for $15 \mathrm{~min}$ at $4{ }^{\circ} \mathrm{C}$. The extract was filtered through $0.45 \mathrm{~mm}$ filters and finally stored at $-20{ }^{\circ} \mathrm{C}$ until use.

In order to prepare the ES products, freshly isolated adult worms $(n \sim 1000)$ were washed four times in normal saline and subsequently in PBS, pH 7.2, containing penicillin $(1000 \mathrm{IU} / \mathrm{ml})$ and streptomycin (1 $\mathrm{mg} / \mathrm{ml}$ ). Worms with high rates of motility were maintained in a sterile culture flask at a density of approximately 100-200 worms/mL of the culture medium (RPMI-1640; Gibco, ThermoFisher Scientific, USA) with penicillin G potassium and streptomycin (1000 IU/ml and $1 \mathrm{mg} / \mathrm{ml})$ and incubated for 24 hours in $5.0 \% \mathrm{CO} 2$ at $37{ }^{\circ} \mathrm{C}$. Supernatants were collected and centrifuged at $12000 \mathrm{rpm}$ for $15 \mathrm{~min}$ at $4{ }^{\circ} \mathrm{C}$. The supernatant was then dialysed against PBS over a period of $24 \mathrm{~h}$, and concentrated using freezedrying process (Lyophilisation) (Zirbus, Netherlands). Protein content was estimated by the Bradford method [21] and stored at $-20^{\circ} \mathrm{C}$ until use.

\section{Preparation of rabbit polyclonal antisera}

Ten adult male rabbits of a commercial New Zealand White strain with average weight of $2 \pm 0.2 \mathrm{Kg}$ were maintained under constant conditions with access to water and food in the animal house, School Veterinary Medicine, Shiraz University. Rabbits were adapted to new conditions for at least two weeks prior to immunization and divided in to 5 groups. Groups 1 to 4 received somatic and ES antigens of $T$. circumcincta, H. contortus, M.marshalli and P. rufescens and group 5 was defined as control. Blood samples (of about $5 \mathrm{ml}$ ) were collected from each rabbit before first injection (day zero) and used as negative controls. According to vaccination protocol (Fig. 1), $300 \mathrm{mg}$ antigen, in a volume of $1 \mathrm{ml}$ of PBS, emulsified with $1 \mathrm{ml}$ of Freund's complete adjuvant (Sigma, USA) was first injected subcutaneously at day zero. This followed by four boosters comprised of $150 \mathrm{mg}$ antigen in a volume of $1 \mathrm{ml}$ of PBS emulsified with $1 \mathrm{ml}$ of Freund's incomplete adjuvant (Sigma, USA). Boosters were given at one week 
intervals. The control group (group 5) was administered with $1 \mathrm{~mL}$ of sterile PBS plus $1 \mathrm{~mL}$ adjuvant. Rabbits were bled one week after final booster (at day 35), sera were collected and stored at $-20^{\circ} \mathrm{C}$ until use. The polyclonal hyper-immune serum was evaluated against somatic and ES antigens by indirect ELISA and Western blotting.

\section{Electrophoretic analysis}

Somatic and ES antigens were separated by sodium-dodecyl-sulfate- polyacrylamide gel electrophoresis (SDS-PAGE) based on Laemmeli's method [22]. The samples were mixed with an equal volume of a sample buffer and boiled for $5 \mathrm{~min}$. They then were added to each well of a $5 \%$ stacking gel and $12 \%$ separating gel. Electrophoresis was run at $100 \mathrm{~V}$ for $4 \mathrm{hr}$ under reducing conditions using electrophoresis apparatus (Paya Pajoohesh Pars, Tehran, Iran). Stained molecular mass standards (Cinnagen PR911654 [SL7012]) ranging from 11 to $180 \mathrm{kDa}$ were used. SDS-polyacrylamide gel was stained for protein visualization with $0.05 \%$ Coomassie brilliant blue (Sigma, USA.).

\section{Western blotting}

For immunoblotting, proteins were first electrophoresed on $12 \%$ SDS-polyacrylamide gel. Western blotting was carried out as described previously [22] with modifications. Proteins were transferred onto a nitrocellulose membrane for $1.5 \mathrm{hr}$. After blocking overnight at room temperature (RT) with $5 \%$ skimmed milk in PBS, nitrocellulose membrane stripes were cut, washed with PBST and incubated with serum sample at RT for $1 \mathrm{hr}$. Sera samples from the immunised rabbits and the infected sheep were diluted as 1:50 and 1:10, respectively. After washing, anti-rabbit and anti-sheep conjugate peroxidase (Sigma, USA) diluted in PBS-T (1:2000 - 1:1000) was added and incubated with shaking for $1 \mathrm{hr}$ at RT. Finally, the membrane stripes was washed and placed into a substrate solution $0.05 \%$ diaminobenzidine in $50 \mathrm{mM}$ Tris $\mathrm{pH} 7.4$ containing $0.05 \% \mathrm{H}_{2} \mathrm{O}_{2}$. (DAB/ $\mathrm{H}_{2} \mathrm{O}_{2}$ ) (Sigma, USA).

The reactivity of sera from sheep and immunized rabbit against $T$. circumcincta somatic and ES antigens was tested against the same products prepared from prevalent field isolates of $H$. contortus, $M$. marshalli and a lungworm species, $P$. rufescens.

\section{Enzyme Linked Immunosorbent Assay (ELISA)}

The checkerboard titration for determination of different dilutions of antigen, sera, and conjugate was done [23]. An indirect ELISA (iELISA) was carried out and optimized with serum samples. 96-well microplates were incubated with $100 \mu \mathrm{l} /$ well of antigen at $1.1 \mathrm{mg} / \mathrm{ml}$ for somatic and $7.5 \mathrm{mg} / \mathrm{ml}$ for ES proteins in $50 \mathrm{mM}$ carbonate bicarbonate buffer $(\mathrm{pH} 9.6)$ at $4^{\circ} \mathrm{C}$ overnight. After washing three times with PBS containing $0.05 \%$ (v/v) Tween 20 (washing buffer), plates were blocked with $200 \mu \mathrm{l} /$ well of the blocking buffer (PBS at pH 7.2 with 1\% Bovine serum albumin) at RT for $2 \mathrm{~h}$. Follow 3 times washings, $100 \mu \mathrm{l}$ of diluted sheep and rabbit sera 1:2 in 1\% BSA were incubated at RT for $1 \mathrm{~h}$. The plates were washed as described above and $100 \mu \mathrm{l} /$ well of horseradish peroxidase anti-sheep and anti-rabbit lgG conjugate (Sigma, USA) diluted at 1:5000 were added and incubated for $1 \mathrm{~h}$ at RT. The plates were 
washed three times and $100 \mu \mathrm{l}$ of the substrate buffer contains ( $0.02 \mathrm{~g}$ Ortho-Phenylenediamine) (Sigma, USA) in citrate buffer and $30 \% \mathrm{H}_{2} \mathrm{O}_{2}$ were added to the plate wells. Finally, the optical density (OD) were obtained from an ELISA reader (Immunoskan BDSL, Thermo Lab. Systems, Finland) at $450 \mathrm{~nm}$. All samples were run in duplicate. A pool containing sera of 10 naturally infected sheep with immunereactivity against $T$. circumcincta (somatic and ES) antigens in western blot test was used as the positive control. Because no serum samples were available from parasite naïve sheep mono-infected with other GINs, the specificity of the method was checked by cross reactivity test using rabbit hyperimmune sera as described earlier.

\section{Statistical analysis}

In order to normalizing the OD estimates in the ELISA, values were quantified as the relative ODR according to the formula: $\mathrm{ODR}=(\mathrm{OD}-\mathrm{N}) /(\mathrm{Ps}-\mathrm{N})$, where $\mathrm{N}$ and $\mathrm{Ps}$ are the mean absorbance values for negative and positive controls. The rates of sensitivity and specificity were evaluated as sensitivity $=$ (true $\mathrm{Ps}) /($ true Ps + false $\mathrm{N}) \times 100$ and specificity $=($ true $\mathrm{N}) /($ true $\mathrm{N}+$ false $\mathrm{Ps}) \times 100$. In order to determine the best cut-off values, receiver operating characteristics (ROC) analysis was performed and the point showing maximum percents of the sensivity and specificity were considered. The SPSS software (Version 16.0) was used for the statistical analyses and the GraphPad Prism 8 for drawing graphs.

\section{Declarations}

\section{Acknowledgments}

The authors thank to Mr. Mlootabi Alavi for his kind technical assistance in the sampling process and the laboratory work.

\section{Authors' contributions}

ER and AY contributed to generate the study plan, data analysis and the manuscript preparation. JA carried out the sample collections and lab work. All authors approved the final draft of the manuscript.

\section{Funding}

This study was supported by a research grant from Shiraz University (No. JA98).

\section{Availability of data and materials}

The nucleotide sequence obtained for the ribosomal ITS region of T. circumcincta was deposited in the GenBank under the Accession number: MN888739 (http://www.ncbi.nlm.nih.gov/nuccore/1955290121). The datasets used/or analysed during the current study are available from the corresponding author on reasonable request.

\section{Ethics approval and consent to participate}


This manuscript is part of a Ph.D. thesis performed by Mr. J. Aliabadi in Shiraz University, Iran. The animal ethics and all protocols were approved by the animal welfare and ethics committee in Faculty of Veterinary Medicine, Shiraz University, Iran (letter No. 983/46/22). All methods were carried out in accordance with Shiraz University animal welfare guidelines and policies. In addition, all the samplings was done when the informed consent was obtained from farm owners.

\section{Consent for publication}

Not applicable.

\section{Competing interests}

The authors declare that they have no competing interests.

\section{Author details}

${ }^{1}$ Department of Pathobiology, School of Veterinary Medicine; Shiraz University, Shiraz, Iran.

\section{References}

1. Lawton DE, Reynolds GW, Hodgkinson SM, Pomroy WE, Simpson HV. Infection of sheep with adult and larval Ostertagia circumcincta: effects on abomasal $\mathrm{pH}$ and serum gastrin and pepsinogen. Int $\mathrm{J}$ Parasitol. 1996;26:1063-1074.

2. Simpson HV, Przemeck SM, Scott I, Pernthaner A. Effects of Teladorsagia (Ostertagia) circumcincta infection on lambs selected for high fleece weight. Vet Parasitol. 2009;165:256-264.

3. Jackson F, Coop RL. The development of anthelmintic resistance in sheep nematodes. Parasitology. 2000;120:95-107.

4. Venturina VM, Gossner AG, Hopkins J. The immunology and genetics of resistance of sheep to Teladorsagia circumcincta. Vet Res Commun. 2013;37:171-181.

5. Miller HRP. Mucosal mast cells and the allergic response against nematode parasites. Vet Immunol Immunopathol. 1996;54:331-336.

6. Stear MJ, Bairden K, Innocent GT, Mitchell S, Strain S, Bishop SC. The relationship between IgA activity against 4th-stage larvae and density dependent effects on the number of 4th-stage larvae of Teladorsagia circumcincta in naturally infected sheep. Parasitology. 2004;29:363-369.

7. Ellis S, Matthews JB, Shaw DJ, Paterson S, McWilliam HEG, Inglis NF, Nisbet AJ. Ovine IgA-reactive proteins from Teladorsagia circumcincta infective larvae. Int J Parasitol. 2014;44:743-750.

8. McRae KM, Stear MJ, Good B, Keane OM. The host immune response to gastrointestinal nematode infection in sheep. Parasite Immunol. 2015;37:605-613.

9. Martínez-Valladares M, Godio-Fernandez R, Vara-Del Rio MP, Martin JF, Rojo-Vazquez FA. Expression of the recombinant protein disulphide isomerase of Teladorsagia circumcincta. Parasite Immunol. 2007;29:47-56. 
10. Johnson DA, Behnke JM, Coles GC. Copro-antigen capture ELISA for the detection of Teladorsagia (Ostertagia) circumcincta in sheep: improvement of specificity by heat treatment. Parasitology. 2004;129:115-126.

11. Malama E, Hoffmann-Köhler P, Biedermann I, Koopmann R, Krücken J, Molina JM, Moreno AM, von Samson-Himmelstjerna G, Sotiraki S, Demeler J. Development of a milk and serum ELISA test for the detection of Teladorsagia circumcincta antibodies in goats using experimentally and naturally infected animals. Parasitol Res. 2014;113:3651-3660.

12. Cruz-Rojo MA, Martinez-Valladares M, Rojo-Vazquez FA. Teladorsagia circumcincta antibodies in serum and milk samples in experimentally infected lactating ewes. Vet Parasitol. 2012;188:386-390.

13. Githiori JB, Kooyman FN, Kruitwagen C, Ploeger HW, Eysker M. Use of a 14.2 kDa recombinant Cooperia oncophora protein in an ELISA for herd health monitoring of nematode infections in first grazing season calves. Vet Parasitol. 2000;91:63-78.

14. Sanchez J, Dohoo IR, Markham F, Leslie K, Conboy G. Evaluation of the repeatability of a crude adult indirect Ostertagia ostertagi ELISA and methods of expressing test results. Vet Parasitol. 2002;109:75-90.

15. Agneessens J, Claerebout E, Vercruysse J. Development of a copro-antigen capture ELISA for detecting Ostertagia ostertagi infections in cattle. Vet Parasitol. 2001;97:229-240.

16. Schallig HD, Hornok S, Cornelissen JB. Comparison of two enzyme immunoassays for the detection of Haemonchus contortus infections in sheep. Vet Parasitol. 1995;57:329-338.

17. Lichtenfels JR, Hoberg EP. The systematics of nematodes that cause ostertagiasis in domestic and wild ruminants in North America: an update and a key to species. Vet Parasitol. 1993;46:33-53.

18. Fakhrahmad F, Rakhshandehroo E, Ghaemi M. Molecular evidence on the emergence of benzimidazole resistance SNPs in field isolates of Marshallagia marshalli (Nematoda: Trichostrongylidae) in sheep. J Parasit Dis. 2020; https://doi.org/10.1007/s12639-020-01319-0.

19. Tamura K, Stecher G, Peterson D, Filipski A, Kumar S. MEGA6: molecular evolutionary genetics analysis version 6.0; 2013.

20. Kimura M. A simple method for estimating evolutionary rates of base substitutions through comparative studies of nucleotide sequences. J Mol Evol. 1980;16:111-120.

21. Bradford MM. A rapid and sensitive method for the quantitation of microgram quantities of protein utilizing the principle of protein-dye binding. Anal Biochem. 1976;72:248-254.

22. Laemmli UK. Cleavage of structural proteins during the assembly of the head of bacteriophage T4. Nature. 1970;227:680-685.

23. Harlow E, Lane D. Antibodies: A Laboratory manual. New York, Cold spring harbor laboratory press; 1988.

24. McNeilly TN, Nisbet AJ. Immune modulation by helminth parasites of ruminants: implications for vaccine development and host immune competence. Parasite. 2014;21:51. 
25. Nisbet AJ, McNeilly TN, Wildblood LA, Morrison AA, Bartley DJ, Bartley Y, Longhi C, McKendrick IJ, Palarea-Albaladejo J, Matthews JB. Successful immunization against a parasitic nematode by vaccination with recombinant proteins. Vaccine. 2013;31:4017-4023.

26. Craig $\mathrm{H}$, Wastling JM, Knox DP. A preliminary proteomic survey of the in vitro excretory/secretory products of fourth-stage larval and adult Teladorsagia circumcincta. Parasitology. 2006;132:535543.

27. Nisbet AJ, Smith SK, Armstrong S, Meikle LI, Wildblood LA, Beynon RJ, Matthews JB. Teladorsagia circumcincta: Activation-associated secreted proteins in excretory/secretory products of fourth stage larvae are targets of early IgA responses in infected sheep. Exp Parasitol. 2010;125:329-337.

28. Redmond DL, Smith SK, Halliday A, Smith WD, Jackson F, Knox DP, Matthews JB. An immunogenic cathepsin F secreted by the parasitic stages of Teladorsagia circumcincta. Int J Parasitol. 2006;36:277-286.

29. Schallig HDFH, Van Leeuwen MAW, Hendrikx WML. Immune responses of Texel sheep to excretorysecretory products of adult Haemonchus contortus. Parasitology. 1994;108:351-357.

30. Molina JM, Ruiz A, Rodriguez-Ponce E, Gutierrez AC, Gonzalez J, Hernandez S. Cross-reactive antigens of Haemonchus contortus adult worms in Teladorsagia circumcincta infected goats. Vet Res. 1999;30:393-399.

31. Gómez-Muñoz MT, Domínguez IA, Gómez-Iglesias LA, Fernández-Pérez FJ, Méndez S, de la Fuente C, Alunda J.M. Cuquerella M. Serodiagnosis of haemonchosis with a somatic antigen $(\mathrm{Hc} 26)$ in several breeds of sheep. J Vet Diagn Invest. 2000;12:354-360.

32. Charlier J, Morgan ER, Rinaldi L, van Dijk J, Demeler J, Höglund J, Hertzberg H, Van Ranst B, Hendrickx G, Vercruysse J, Kenyon F. Practices to optimise gastrointestinal nematode control on sheep, goat and cattle farms in Europe using targeted (selective) treatments. Vet Rec. 2014;175:250255.

\section{Figures}




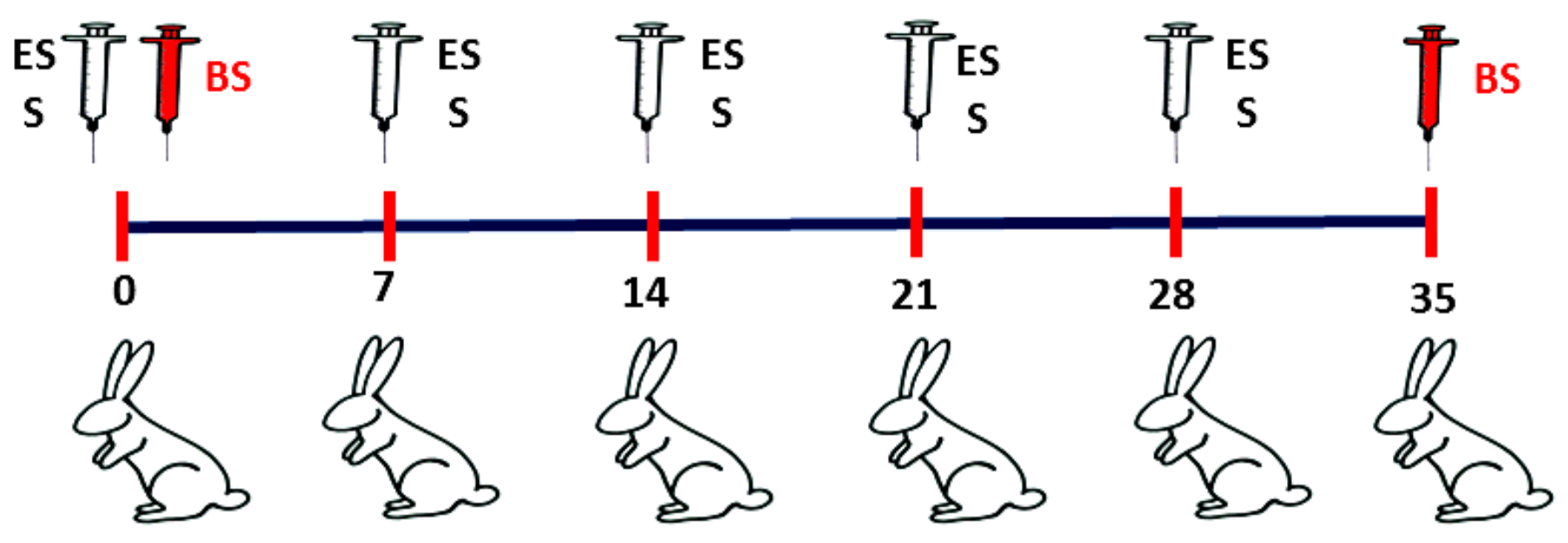

Figure 1

Vaccination protocol for immunization of rabbits against different (somatic(S) and ES) antigenic materials and the respective times for blood sampling (BS).

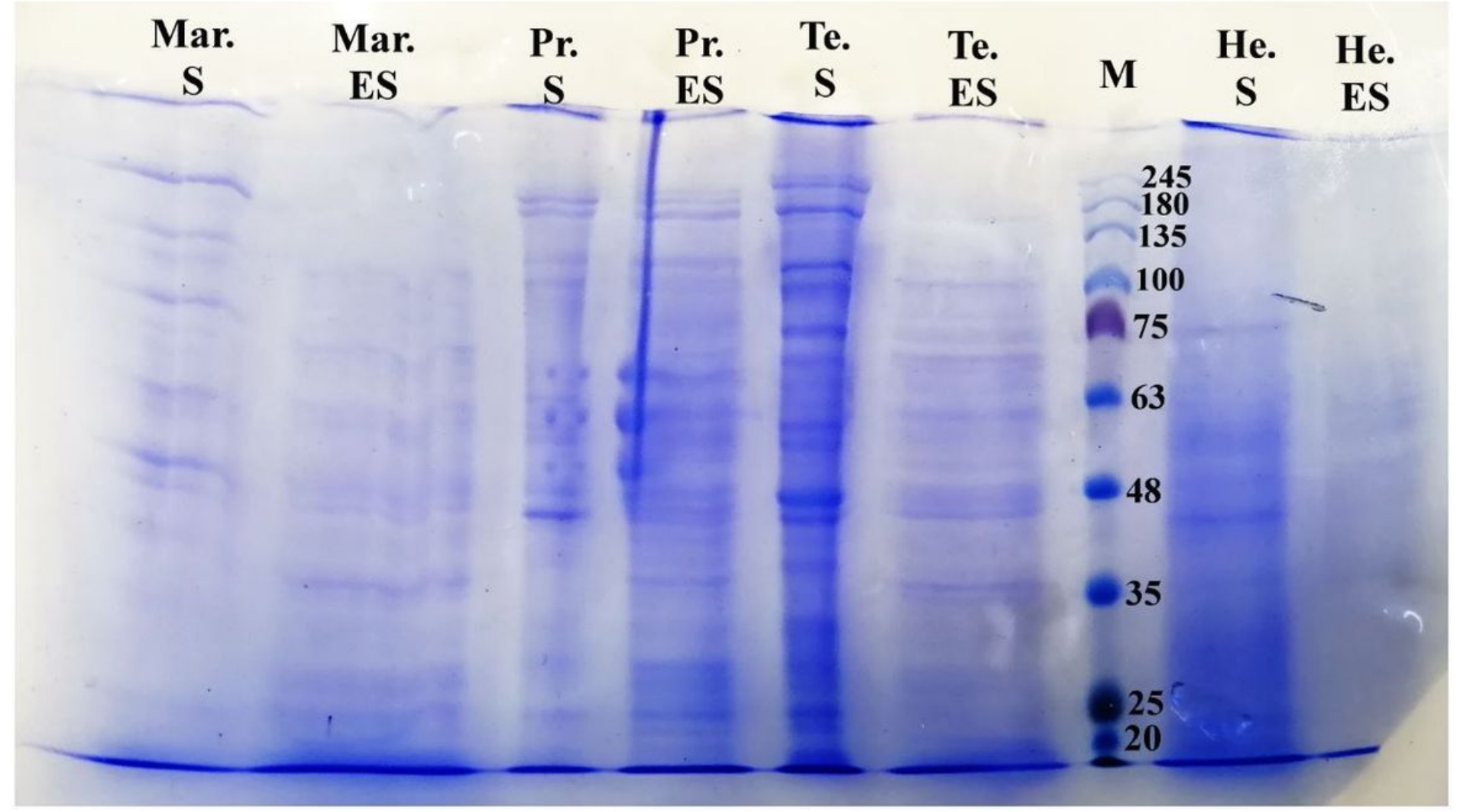

Figure 2 
SDS-PAGE analysis of somatic and ES antigens for adult stages of T. circumcincta, H. contortus, P. rufescens and M. marshalli; M: Protein molecular weight marker.

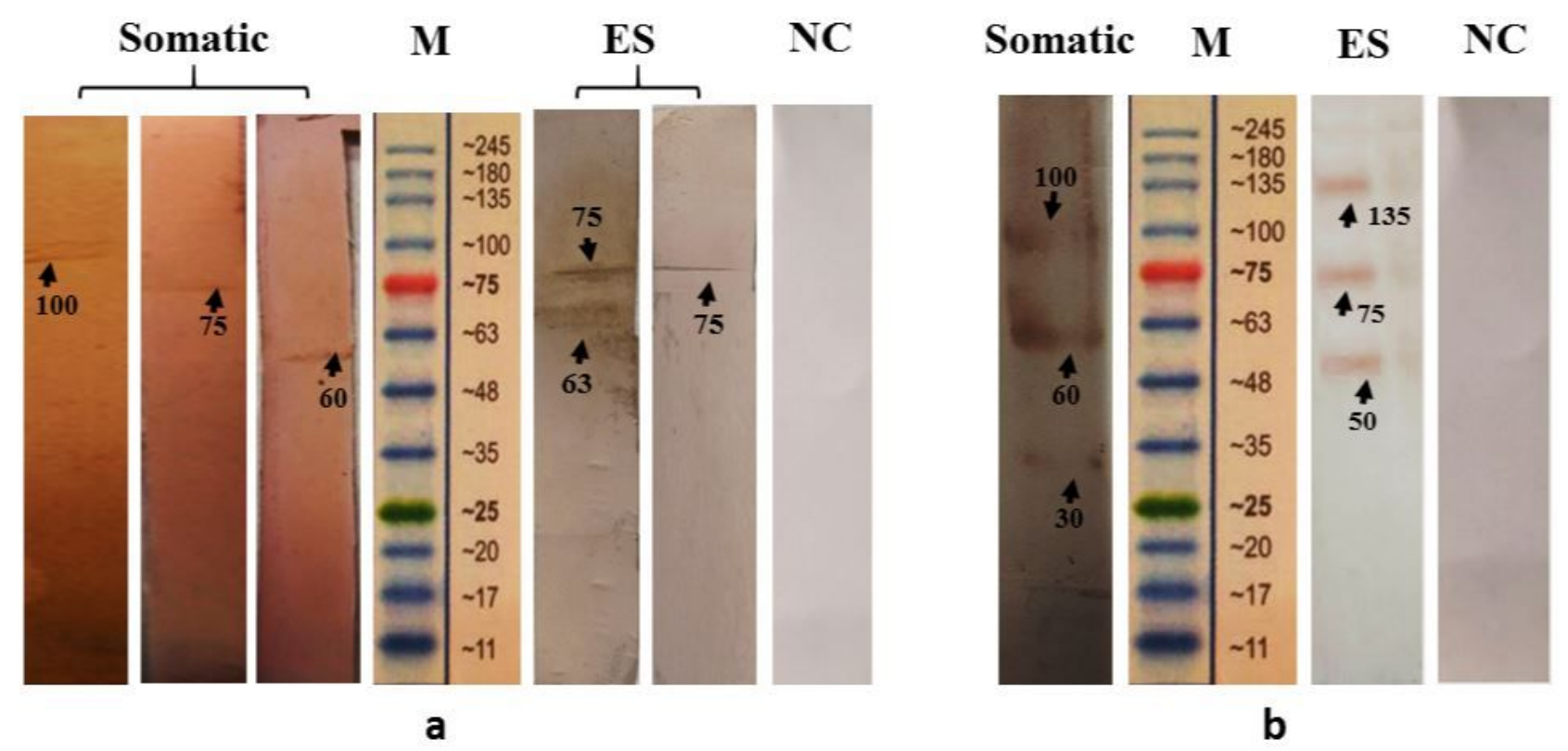

\section{Figure 3}

Comparative western blot analysis of immunodominant proteins in somatic and ES content reactive to sera from the T. circumcincta infected sheep (A) and from hyper immune sera raised in rabbits (B). NC: non- immunized rabbit as negative control; M: Protein molecular weight marker. 


\section{Somatic}

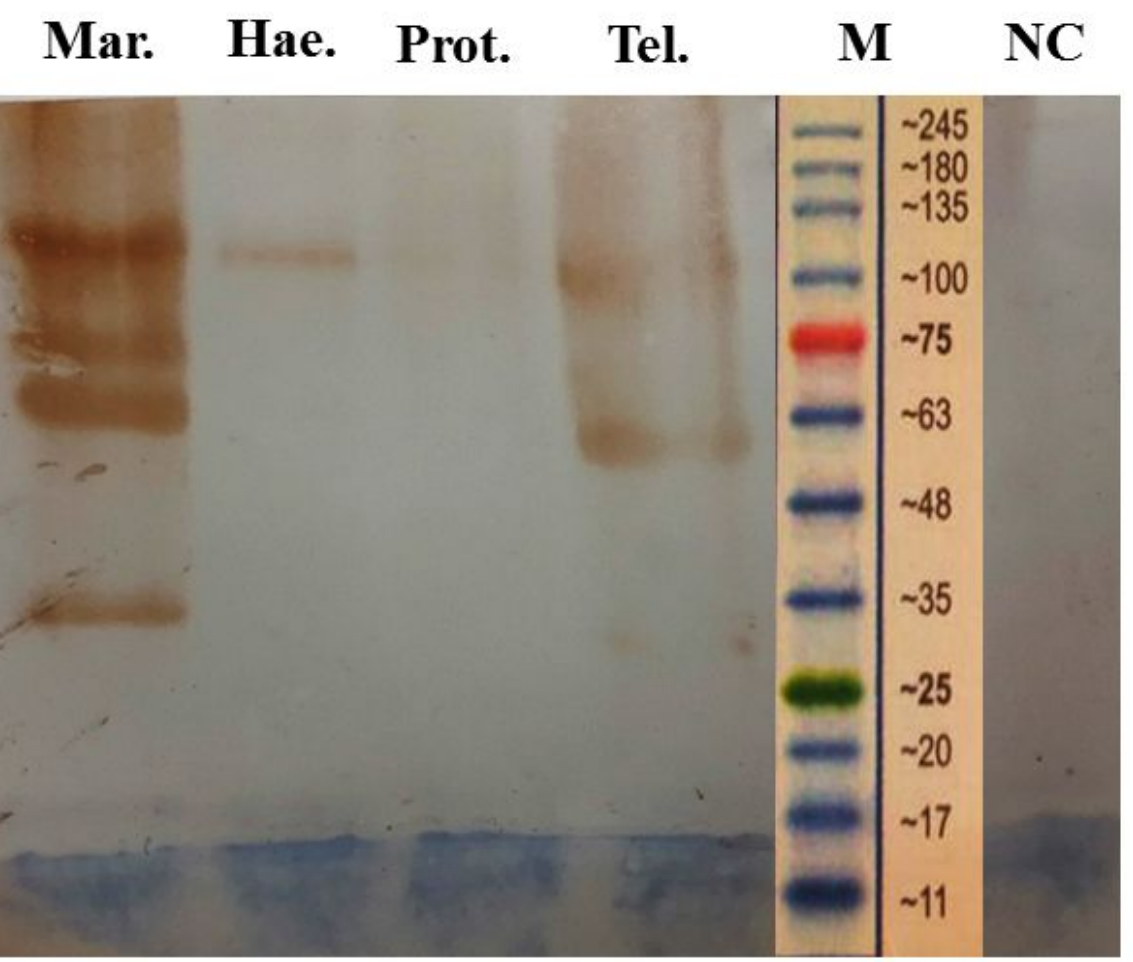

ES

Mar. Hae. Prot.
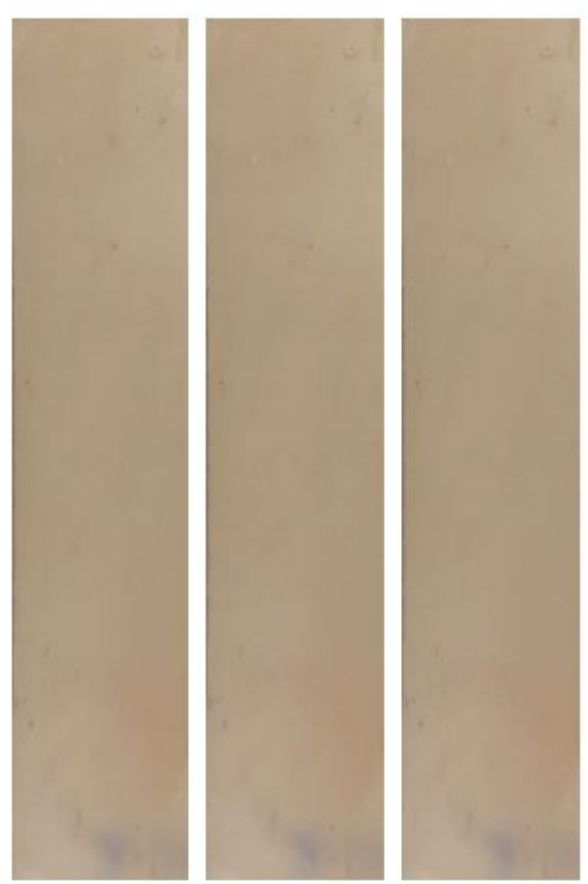

Figure 4

Western blot analysis. Cross reactivity of rabbit hyper immune sera against somatic and ES antigens of $\mathrm{P}$. rufescens, $\mathrm{H}$. contortus and $\mathrm{M}$. marshalli. NC: non- immunized rabbit as negative control; M: Protein molecular weight marker. 


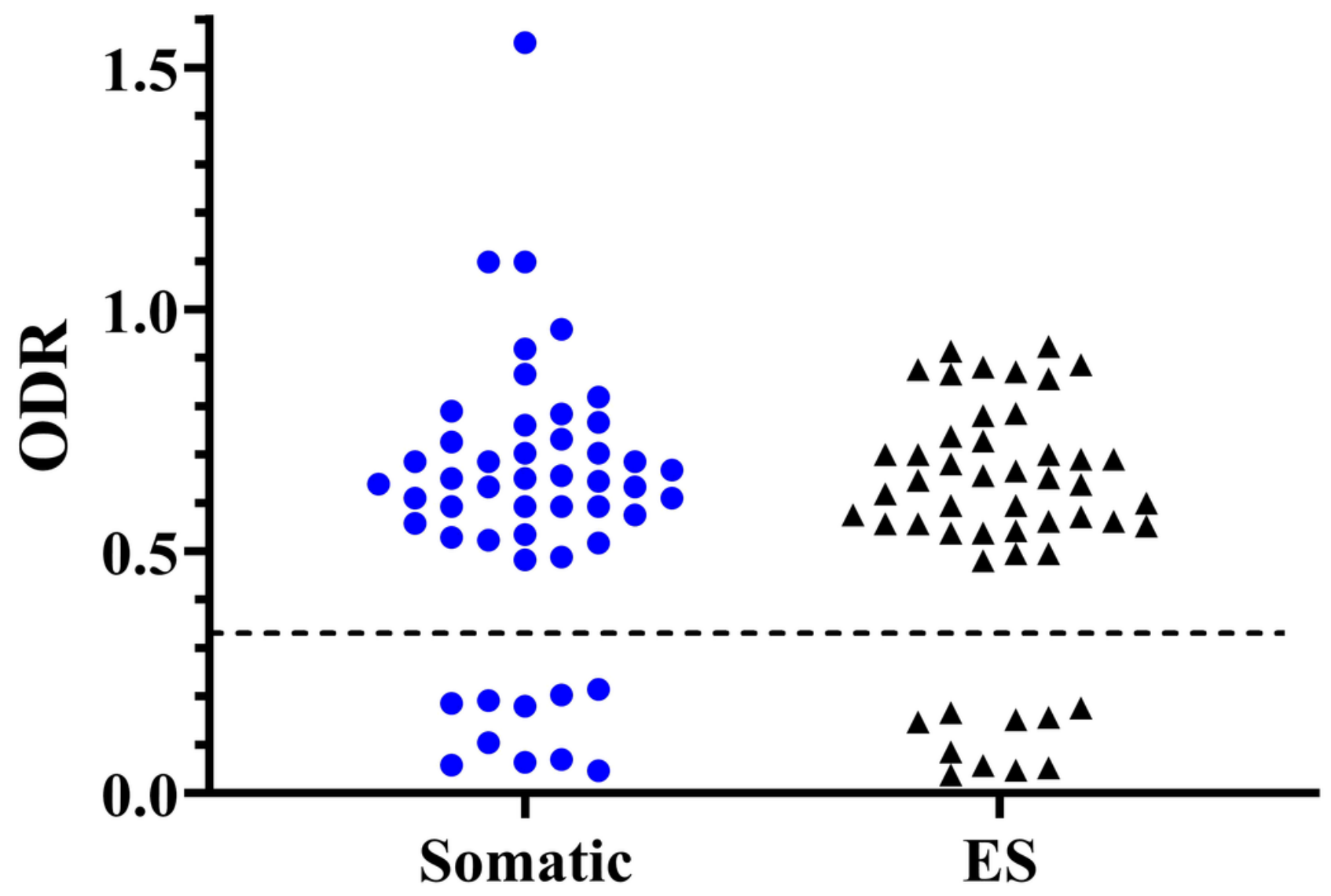

Figure 5

Optical density ratio (ODR) values obtained from sera samples of positive (T. circumcincta infected) and negative (non-infected) controls (NC) against somatic and ES products. The dashed line shows the approximate value of estimated cut-off value. Samples under the line are representative of negative controls and those above the line relates to positive ones. 


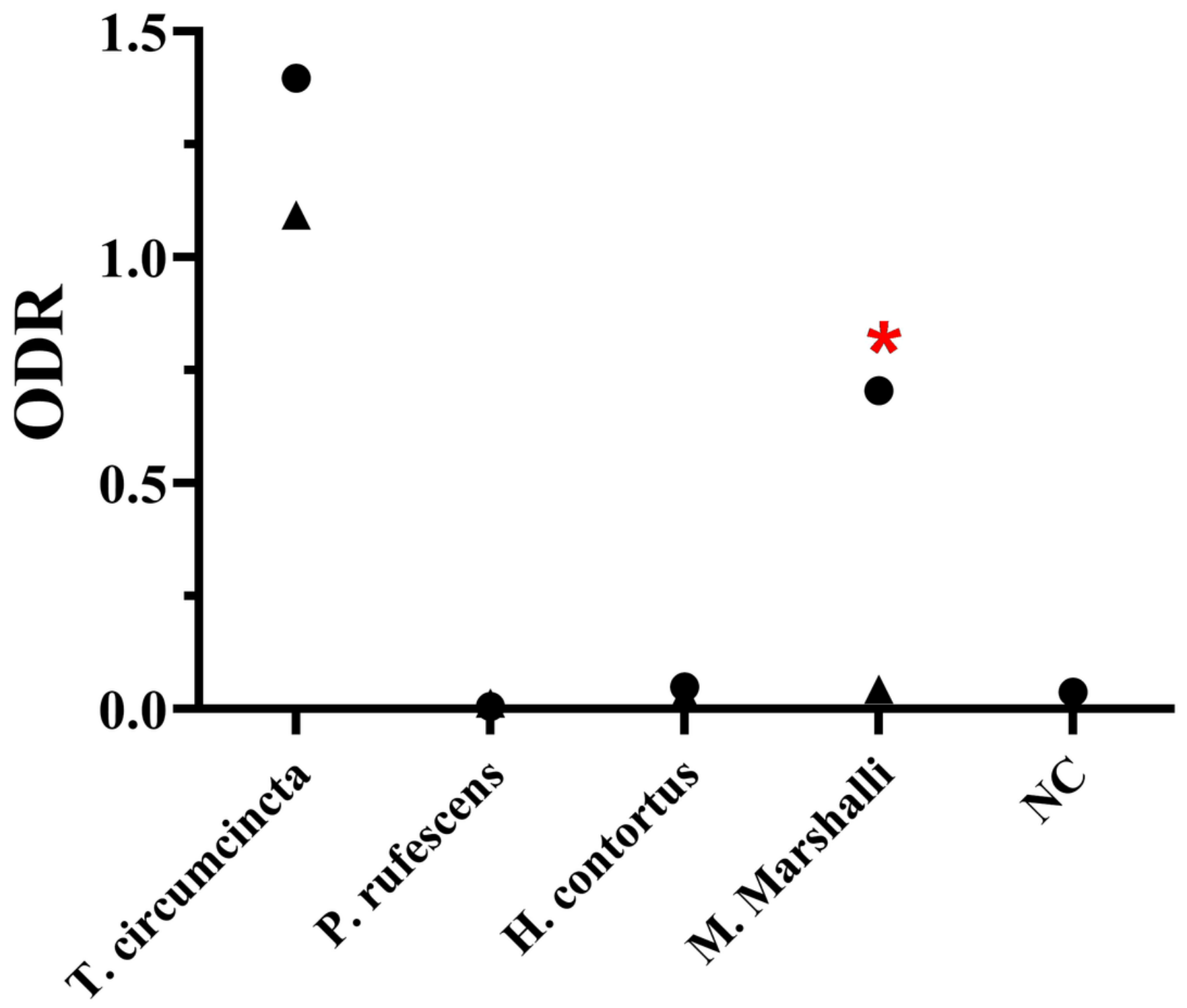

Figure 6

Cross-reactivity by ELISA method. The optical density ratio (ODR) values obtained from rabbit hyperimmune sera against somatic and ES of different prevalent worms. 